Revista em Agronegócio e Meio Ambiente, Maringá (PR)

DOI: http://dx.doi.org/10.17765/2176-9168.2018v11n2p663-681

\title{
INDICAÇÃO GEOGRÁFICA E ATRIBUTOS DAS REGIÕES DE ORIGEM NO COMPORTAMENTO DO CONSUMIDOR DE VINHOS: UMA REVISÃO SISTEMÁTICA DA LITERATURA
}

\author{
Luis Henrique Ramos Camfield* \\ Jean Philippe Palma Révillion** \\ Saionara Araujo Wagner ${ }^{* * *}$ \\ Carla Mecca Giacomazzi***** \\ Liris Kindlein ${ }^{* * * * *}$
}

RESUMO: O objetivo deste trabalho foi compreender as variáveis que interferem na percepção de qualidade dos consumidores de vinhos com indicação geográfica ou com atributos regionais. Foi realizada, para tanto, uma revisão sistemática, a partir de estudos publicados em periódicos indexados nas bases de dados online Web of Science, Scopus e Science Direct, com a seguinte estratégia de busca: ((wine) AND (consumerbehav*)). Nesse contexto, foram procuradas informações relacionadas ao consumidor de vinhos com indicação geográfica, considerando-se denominação de origem, indicação de procedência ou ainda, relacionadas a atributos regionais. A abordagem metodológica de revisão sistemática mostrou-se adequada para a realização de uma revisão capaz de apresentar diferentes visões sobre o comportamento dos consumidores de vinhos. Verificou-se que atributos intrínsecos, como sabor ou aroma, e extrínsecos, como a embalagem e rótulo, influenciam na decisão de compra dos consumidores de vinhos com indicação geográfica ou com atributos regionais. Os resultados demonstram que os consumidores de vinhos com indicação geográfica ou com atributos regionais vêm valorizando a qualidade sensorial, tipicidade e prestígio dos vinhos, proporcionando maior credibilidade aos produtos. Além disso, os atributos intrínsecos e extrínsecos parecem estar relacionados e fortemente associados a uma determinada região, influenciando na decisão de compra do consumidor.

\footnotetext{
Docente Dr. Instituto Federal de Educação, Ciência e Tecnologia do Rio Grande do Sul (IFRS), Brasil. E-mail: luis.camfield@bento.ifrs.edu.br

** Docente Dr. Programa de Pós-graduação em Agronegócios, Centro de Estudos e Pesquisas em Agronegócios (CEPAN), Universidade Federal do Rio Grande do Sul (UFRGS), Brasil.

*** Docente Dr ${ }^{\mathrm{a}}$. Faculdade de Veterinária da Universidade Federal do Rio Grande do Sul (UFRGS), Brasil.

${ }^{* * * *}$ Mestre em Agronegócios pelo Centro de Estudos e Pesquisas em Agronegócios da Universidade Federal do Rio Grande do Sul (UFRGS) (CEPAN), Universidade Federal do Rio Grande do Sul (UFRGS), Brasil.

***** Docente Dra ${ }^{\mathrm{a}}$. Programa de Pós-graduação em Agronegócios, Centro de Estudos e Pesquisas em Agronegócios (CEPAN), Universidade Federal do Rio Grande do Sul (UFRGS), Brasil.
} 
PALAVRAS-CHAVE: Vinhos; Percepção do consumidor; Produção local; Atributos intrínsecos e extrínsecos.

\title{
GEOGRAPHIC INDICATION AND ATTRIBUTES OF REGIONS OF ORIGIN WITH REGARD TO BEHAVIOR OF WINE CONSUMERS: A REVIEW OF THE LITERATURE
}

\begin{abstract}
Variables that interfere in the quality perception of wine consumers with regard to geographic indications or regional attributes are analyzed. A database review was undertaken, foregrounded on studies published in indexed journals Web of Science, Scopus and Science Direct, with the following search strategy: ((wine) and (consumerbehav*)). Information on the relationship between wine consumers and geographic indication, as region of origin, source, or even regional attributes was provided. Methodological approach proved to be adequate for the review and was able to provide different views on wine consumers' behavior. Intrinsic attributes, such as taste and aroma, and extrinsic ones, such as labels and packing, featuring geographic indications or regional attributes, affect purchase by wine consumers. Results show that consumers of wines with geographic indications or regional attributes enhance the sensorial quality, type and prestige of wines, through a greater credibility in the products. Further, intrinsic and extrinsic attributes seem to be related and strongly associated to a specific region and, consequently, influence the consumer's decision.
\end{abstract}

KEY WORDS: Wines; Consumers' perception; Local production; Intrinsic and extrinsic attributes.

\section{INTRODUÇÃO}

As atividades vitivinícolas possuem destacada importância no agronegócio mundial, pois, de acordo com relatório da Food and Agriculture Organization (FAO, 2010), a produção de vinho utiliza como matéria-prima a uva, que está entre os alimentos processados mais importantes no cenário mundial, em paralelo a alimentos como cevada, sementes de algodão, azeite de oliva e óleo de milho, entre outros. Estudos já realizados sobre o tema comportamento do consumidor de vinhos, particularmente aqueles que apresentam algum tipo de certificação 
(KRYSTALLIS; CHRYSOCHOU, 2009; THIENE et al., 2013), destacaram a importância da percepção e lealdade do consumidor para o desenvolvimento de estratégias de mercado, principalmente relacionadas à produção local e à certificação de origem.

Pesquisas que tratam da produção de vinhos vêm discutindo aspectos relacionados às questões de valorização de produção local ou regional e certificações. Nesse sentido, a compreensão do comportamento do consumidor de alimentos certificados tem se tornado fundamental para os pesquisadores e empresas, uma vez que este comportamento se mostra dinâmico e instável, uma vez que está sujeito a variáveis ambientais e mercadológicas capazes de influenciar na intenção de compra. A complexidade existente nesse fator exige que o comportamento do consumidor seja estudado e monitorado a fim de se estabelecer novas estratégias de marketing (HAWKINS; MOTHERSBAUGH, 2012).

Nesse sentido, o comportamento dos consumidores de vinhos, em particular, é influenciado por diferentes fatores que estão associados à qualidade, como os atributos sensoriais, a confiabilidade e a credibilidade estabelecida entre o consumidor e a vinícola. Assim, as certificações dos vinhos se destacam como um elemento capaz de conduzir uma boa estratégia de comercialização, tornando esses produtos mais competitivos (ROSSI, 2009; THIENE et al., 2013). Como diversos estudos apontam que a percepção de qualidade dos vinhos para os consumidores é determinada pelos seus atributos, faz-se necessário compreender quais são essas características, e como elas interferem na decisão de compra (ROMA et al., 2013; CORDUAS et al., 2013).

As indicações geográficas de vinhos (denominação de origem ${ }^{6}$, indicação de procedência ${ }^{7}$ ou equivalentes) estão sendo utilizadas nas últimas décadas como um atributo de confiança na hora de decisão da compra dos vinhos, principalmente na Europa, onde esse processo já está difundido há mais tempo. Os

6 Denominação de origem corresponde ao nome geográfico de país, cidade, região ou localidade de seu território, que designe produto ou serviço, cujas qualidades ou características se devam exclusiva ou essencialmente ao meio geográfico, incluídos fatores naturais e humanos (INPI, 2015).

7 Indicação de procedência é o nome geográfico de um país, cidade, região ou uma localidade de seu território, que se tornou conhecida como centro de produção, fabricação ou extração de determinado produto ou prestação de determinado serviço (INPI, 2015). 
vinhos com atributos regionais, ${ }^{8}$ embora não tenham recebido certificação formal, aparecem frequentemente na literatura internacional (AZABAGAOGLU et al., 2006; MILOHANOVIĆ; BRŠČIĆ, 2008; JAEGER et al., 2009; CHREA et al., 2011; ATKIN; THACH, 2012; BERNABÉU et al., 2013; BRUWER; BULLER, 2013; KALLAS et al., 2013; BRUWER et al., 2014).

Os atributos regionais transmitem confiabilidade para os consumidores, já que são associados à região de origem do vinho com atributos de qualidade. $\mathrm{Na}$ Argentina, por exemplo, os consumidaores de vinho sabem que os vinhos procedentes de Mendoza podem ser considerados bons, pois esta é uma região que possui atributos de solo e clima propícios à produção de uvas de boa qualidade.

$\mathrm{Na}$ percepção do consumidor de que a qualidade do vinho pode estar associada aos selos de indicação geográfica destacam-se tanto os atributos intrínsecos como também os extrínsecos relacionados ao prestígio e à credibilidade das vinícolas ou dos produtores da região delimitada (OLSON; JACOBY, 1972; SCHIFFMAN, 1997). Assim, os selos de indicação geográfica podem resultar na definição de atributos que estejam associados a uma identidade dos vinhos de uma determinada região, podendo levar à fidelização do consumidor (PERROUTY et al., 2006).

Estudos demonstram ainda que atributos extrínsecos, como rótulo, embalagem, rolha, marca e estilo, também influenciam na decisão de compra dos consumidores. A embalagem, branding e rotulagem se destacam neste quesito e exercem boa influência no momento da compra (MUELLER; SZOLNOKI, 2010; CORDUAS et al., 2013; GARCIA-GALÁN et al., 2014; SAÉNZ-NAVAJAS, 2014).

Assim, a análise das percepções dos consumidores potenciais de vinhos com indicação geográfica ou com atributos regionais a partir de uma revisão sistemática pode auxiliar na compreensão das variáveis que interferem na decisão de compra, bem como auxiliar as empresas que produzem vinhos a definirem seus planos estratégicos.

8 Atributos regionais de caráter informal referem-se aos casos em que o produto tem o nome da região de produção, cujas características de qualidade decorrem dos atributos desse território (solo, clima) e do saber fazer (tradição) dos produtores, com tipicidade única; porém, ainda não receberam algum tipo de certificação. 


\section{MAERIAL E MÉTODOS}

Este estudo foi baseado na elaboração de uma revisão sistemática, por meio de um levantamento de estudos que são relevantes ao tema. A revisão bibliográfica sistemática foi definida por Greenhalgh (1997) "como uma síntese de estudos primários que contém objetivos, materiais e métodos claramente explicitados e que foi conduzida de acordo com uma metodologia clara e reprodutível”. Já para Colicchia e Strozzi (2012), a revisão sistemática da literatura propicia a identificação dos artigos mais relevantes a serem incluídos no estudo, a fim de revelar a dinâmica do campo de estudo. As informações foram recolhidas e sintetizadas com publicações ocorridas entre o ano de 2004 e o ano de 2014, com busca nas bases de dados online Isi Web of Science, Scopus e Science Direct, com a seguinte estratégia de busca: (wine AND consumer behav*).

$\mathrm{Na}$ fase inicial de seleção de estudos, mediante avaliação do título e resumo, os trabalhos foram selecionados de acordo com os seguintes critérios: i) constituição de pesquisa primária ou de revisão; ii) avaliação do perfil do consumidor de vinhos; iii) trabalhos descritivos (survey ou observação) ou realização de painel/ avaliação/análise sensorial; iv) abordagem de certificações de indicação geográfica, denominação de origem, indicação de procedência ou estudos com atributos de origem. A partir da definição da questão de pesquisa, que buscou identificar os estudos referentes às indicações geográficas de vinhos, três pesquisadores avaliaram, analisaram e selecionaram os estudos que tratavam do tema. Foram analisados os dados e interpretados os resultados dos estudos selecionados. Na Figura 1 é apresentado um fluxograma que demonstra a estratégia de busca nos portais e a avaliação dos artigos realizada posteriormente, demonstrando a sequência das etapas para seleção, avaliação e interpretação dos resultados. 


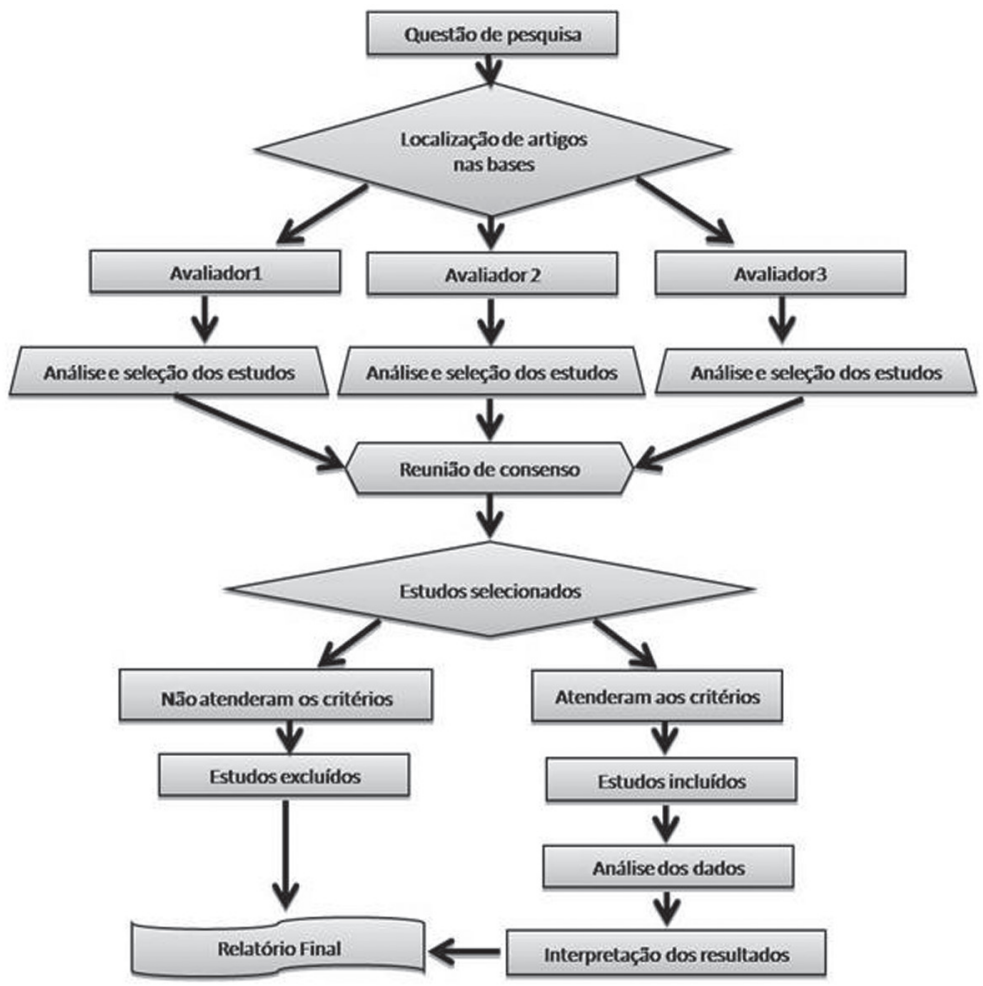

Figura 1. Fluxograma da estratégia de busca dos artigos para revisão sistemática Fonte: Adaptado de Urso (2011)

A estratégia de busca e tratamento apresentados na Figura 1 não aplicou tratamento algum aos dados apresentados nos estudos selecionados, somente executou um levantamento de dados dentro do universo disponível - neste caso, consumidor de vinhos certificados ou com referência a atributos de origem. A organização dos dados dos artigos e toda a análise foram realizadas em planilhas do Microsoft Excel e no editor de texto Microsoft Word. 


\section{RESULTADO E DISCUSSÃO}

$\mathrm{Na}$ elaboração da revisão sistemática foram obtidos, inicialmente, 487 trabalhos, sendo que, após leitura e avaliação, 29 foram aprovados e 458 foram descartados em função de não se enquadrarem nos critérios propostos. Após reavaliação mais detalhada dos artigos, mais quatro foram descartados, restando 25 artigos completos. No Quadro 1são apresentados os principais dados descritivos dos artigos utilizados na revisão sistemática publicada nos portais ISI Web of Science, Scopus e Science Direct, cujo tema abrangia estudos sobre o comportamento do consumidor de vinhos com foco em indicações geográficas e atributos de origem. 


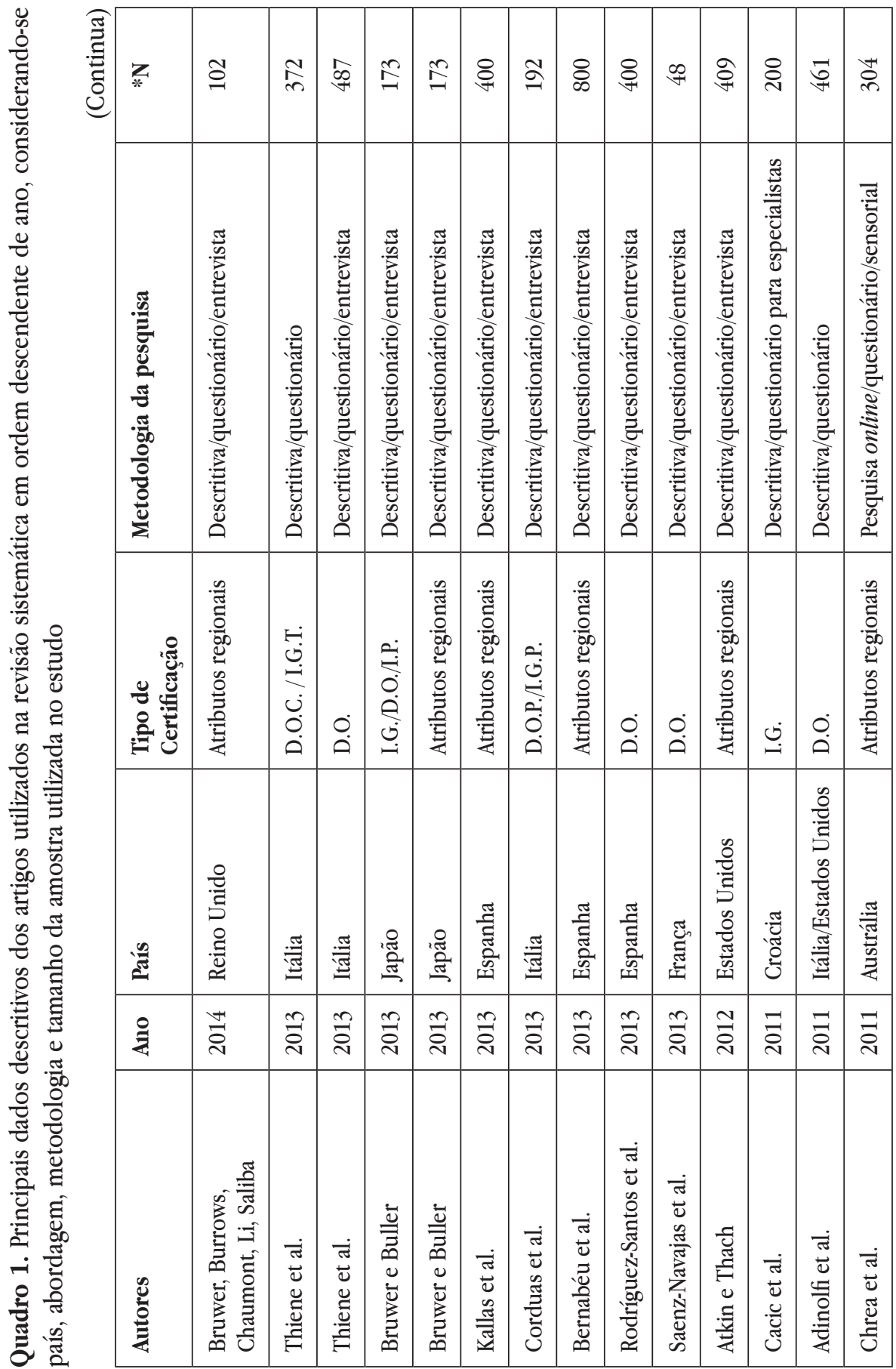




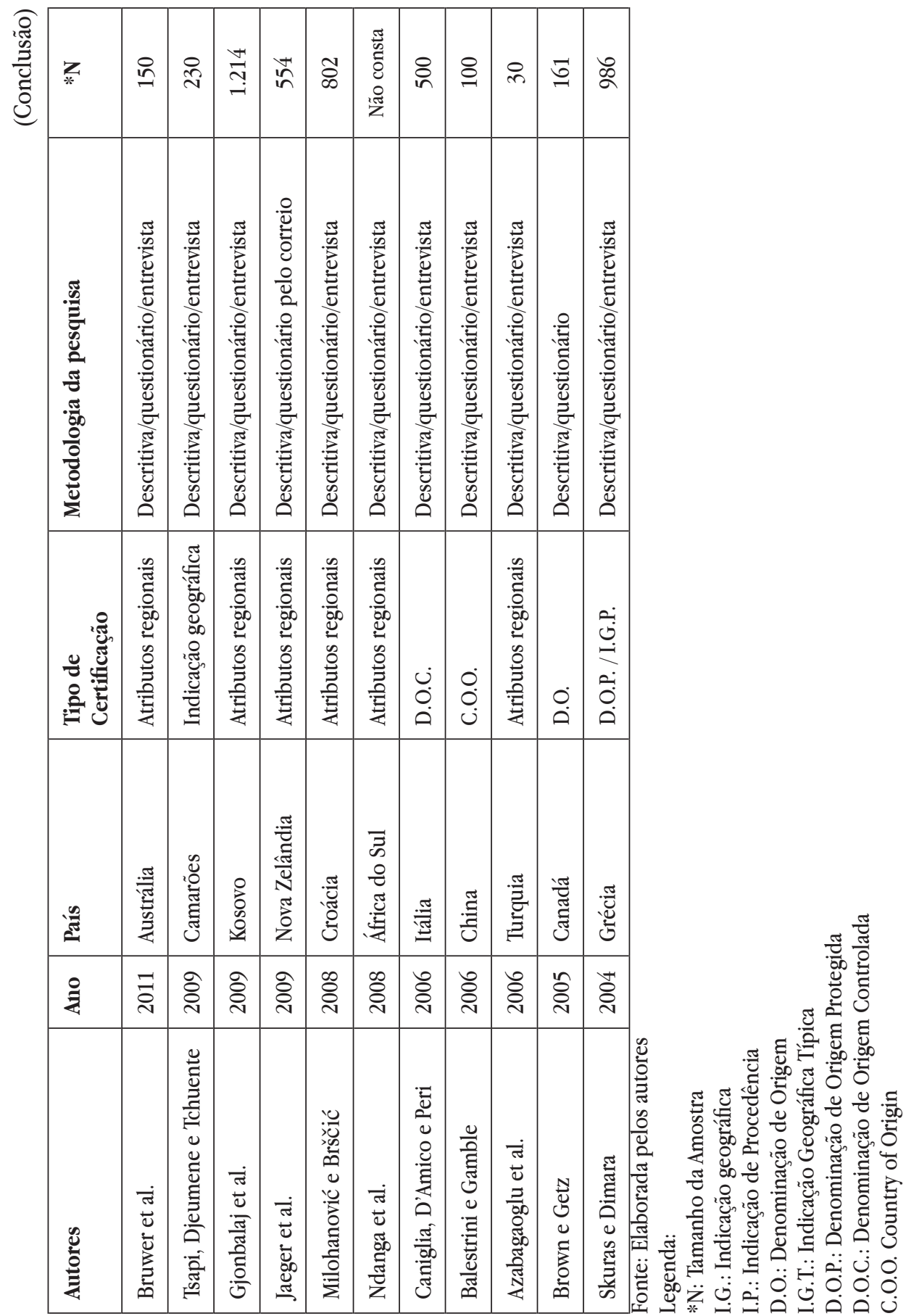


Foi realizada uma busca sobre os períodos disponíveis, nas bases selecionadas, para todos os períodos à disposição e os resultados apresentaram estudos ocorridos no período entre 2004 e 2014, o que demonstra que o tema vem sendo pesquisado há pouco tempo. Isso ocorre possivelmente porque, somente no ano de1995, as indicações geográficas passaram a ser protegidas internacionalmente no âmbito da Organização Mundial do Comércio, reguladas pelos artigos 22 a 24 do Acordo TRIPS. ${ }^{9}$ As pesquisas associadas com o tema, os respectivos continentes de realização e o número de estudos sobre indicação geográfica ou região de origem são apresentados na Figura 2.

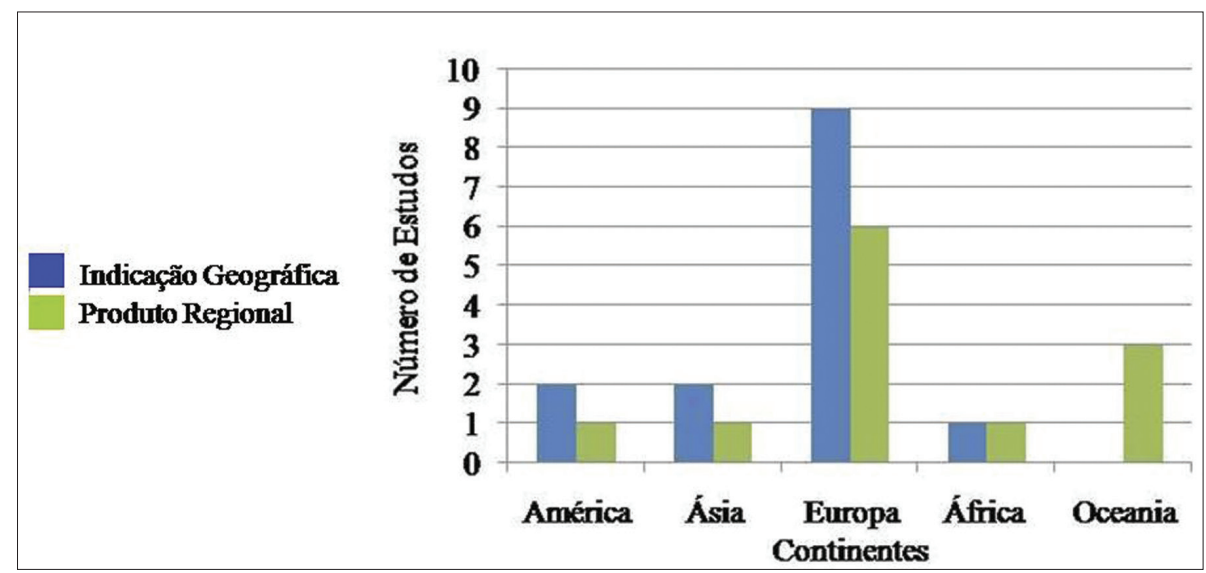

Figura 2. Continentes onde foram realizadas as pesquisas e tipo de estudo Fonte: Elaborada pelos autores

Com relação à distribuição espacial, observou-se que houve maior concentração de pesquisas no continente europeu (57,69\%), seguido pelas Américas (11,54\%), Ásia (11,54\%), Oceania (11,54\%) e África (7,69\%). Quanto ao tipo de certificação avaliada, evidenciou-se a predominância da certificação geográfica (53,85\%), seguida por produto regional (46,15\%). É importante destacar que o estudo realizado na Turquia foi considerado para os continentes asiático e europeu, por ser este um país euroasiático.

9 TRIPS (Trade-RelatedAspects of IntellectualPropertyRights) é um acordo que foi firmado em 1994 com o intuito de: a) minimizar as distorções e obstáculos ao comércio internacional e levando em consideração a necessidade de promover uma proteção eficaz e adequada dos direitos de propriedade intelectual e b) assegurar que as medidas e procedimentos destinados a fazê-los respeitar não se tornem, por sua vez, obstáculos ao comércio legítimo. 
A maior concentração de pesquisas realizadas em países europeus pode ser explicada por sua tradição na produção e comercialização de vinhos com certificações para o mercado internacional, destacando-se países com maior tradição, como Itália, França, Portugal e Espanha (OIV, 2014). Esses países destacamse internacionalmente e, para tanto, têm investido na produção e comercialização de vinhos, usando estratégias de mercado com ênfase na certificação e valorização de atributos regionais.

Não foram encontrados estudos, nas bases de dados pesquisadas sobre esse tema, referentes aos países da América do Sul, embora países como Argentina, Chile e Brasil vêm produzindo vinhos de boa qualidade e obtendo selos de indicações de geográficas. Isso ocorreu possivelmente porque esses países têm investido em certificações há pouco tempo, se comparados com países europeus. Além disso, os resultados são limitados pela estratégia de busca, o que restringiu os estudos incluídos nessa pesquisa. Em relação a isso, poderia se utilizar outra estratégia de busca, com diferentes palavras-chave, a fim de ampliar o número de estudos obtidos, incluindo pesquisas realizadas na América do Sul. Assim seria possível cobrir uma nova lacuna de pesquisa, inclusive poderia se identificar diferenças e/ ou semelhanças entre os resultados dos estudos.

Quanto ao tipo de estudo dos artigos selecionados, ficou destacada a maior utilização de metodologias descritivas do que sensoriais. Nos 25 estudos utilizados nessa revisão, participaram 9.248 consumidores, sendo que, desses, 8.896 foram entrevistados para estudos descritivos e 352 participaram de análises sensoriais, evidenciando que a maioria privilegiou a utilização de metodologias descritivas baseadas na aplicação de questionários.

Nos artigos selecionados percebe-se que os consumidores de vinhos com certificação demonstraram valorizar mais os atributos extrínsecos, considerando, principalmente, fatores como rótulo, embalagem, disponibilidade do produto, preço, estética do produto, marca, promoções e estilo (CHREA et al., 2011; GARCIAGALAN et al., 2014; SAENZ-NAVAJAS et al., 2014), utilizando tanto uma abordagem qualitativa, quanto quantitativa nos instrumentos de coleta de dados.

Observa-se que os fatores intrínsecos e extrínsecos, que estão fortemente relacionados com as certificações de origem, possuem impacto semelhante no 
comportamento de compra dos consumidores, independentemente do local onde foram realizados os estudos. Esses atributos são considerados decisivos para os consumidores, pois, de acordo com Albersmeier et al.(2009) e Alebaki e Iakovidou (2010), o nível de exigência quanto à qualidade e segurança alimentar dos produtos tem aumentado. Assim as vinícolas poderão buscar alternativas e criar estratégias para ampliar o atendimento aos consumidores que buscam produtos com indicação geográfica, com vistas a ampliar o consumo e, consequentemente, o mercado desses vinhos.

Os resultados apresentados nos artigos selecionados indicaram, ainda, que os fatores extrínsecos são determinantes na escolha do produto. Neste caso, os consumidores consideram o preço um atributo-chave para a escolha do vinho, sendo, frequentemente, relacionado à qualidade do produto (BERNABÉU et al., 2013), marca (NDANGA et al., 2008), imagem e reputação do vinho (BALESTRINI; GAMBLE, 2006; CANIGLIA et al., 2008; NDANGA et al., 2008; CACIC et al., 2011; CHREA et al., 2011; THIENE et al., 2013; SAENZ-NAVAJAS et al., 2014). Além disso, a safra ou estilo, associada à qualidade do vinho, também foi considerada importante (SAENZ-NAVAJAS et al., 2014).

Em seus estudos, Chrysochou et al.(2012); Priilaid e Van Rensburg (2012); Corduas et al.(2013) destacaram que os atributos intrínsecos do vinho também são considerados essenciais na decisão de compra dos consumidores, sugerindo qualidade e confiabilidade, evidenciadas mais fortemente nos consumidores italianos, em que a embalagem e a etiqueta têm pouca importância, pois os consumidores com maior conhecimento sobre vinhos valorizam mais fatores intrínsecos (CORDUAS et al., 2013). O comportamento italiano, no quesito do consumo, é semelhante ao dos consumidores japoneses, que apresentam níveis mais elevados de conhecimento objetivo sobre vinhos, considerando as pistas intrínsecas mais importantes do que as extrínsecas para a decisão de compra (BRUWER et al., 2012).

Os artigos que tratam dos atributos de origem do vinho, mesmo que sem certificação (AZABAGAOGLU et al., 2006; MILOHANOVI ; BRŠ I, 2008; ATKIN; THACH, 2012; BERNABÉU et al., 2013; KALLAS et al., 2013), apresentaram resultados que convergem com pesquisas de indicação geográfica, ou seja, consideram a região onde o vinho foi produzido como um forte indicativo de qualidade. Além disso, os estudos analisados demonstraram que há relação dinâmica entre os fatores extrínsecos, os intrínsecos e o comportamento dos consumidores de vinhos, conforme pode ser observado na Figura 3. 


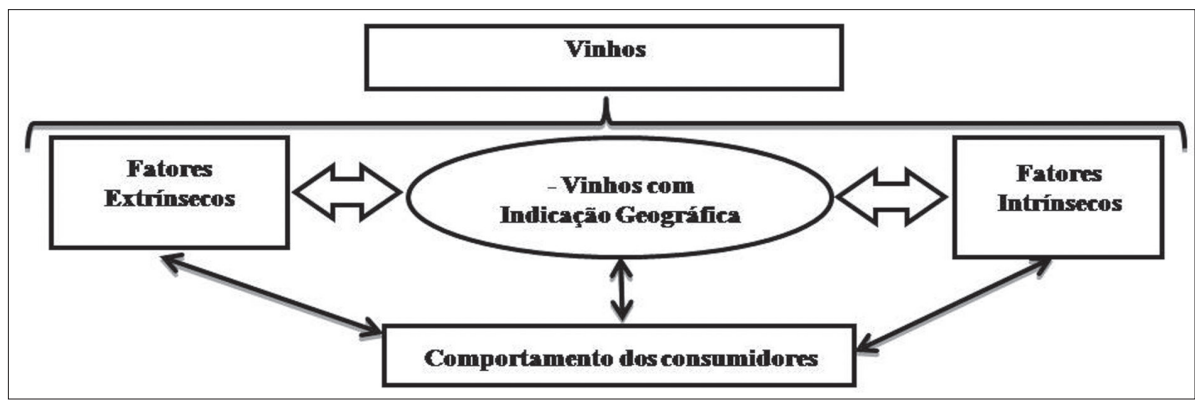

Figura 3. Relação entre fatores intrínsecos e extrínsecos e comportamento dos consumidores de vinhos

Fonte: Elaborado pelos autores

Os fatores intrínsecos e extrínsecos influenciam o comportamento dos consumidores e estão interligados e fortemente relacionados com as certificações de origem e, estas, com a qualidade. Cabe salientar, também, que, nos artigos selecionados, apesar da existência das certificações de origem, o comportamento dos consumidores de vinhos varia em função do nível de conhecimento e envolvimento com o produto (BRUWER et al., 2014).

Verificou-se que os atributos intrínsecos dos vinhos são fatores determinantes para o desenvolvimento de uma indicação geográfica, ou seja, a partir de características próprias de determinada região poderá ser obtida a indicação geográfica (IG), capaz de atestar a tipicidade e a procedência do vinho. No caso dos vinhos com atributos regionais, a associação parece ser a mesma, pois, de acordo com as características próprias de determinada região, o vinho será reconhecido e valorizado, mesmo que não tenha certificação de origem formalmente emitida.

\section{CONSIDERAÇÕES FINAIS}

Pode-se concluir que houve aumento no número de pesquisas e publicações que tratam da temática do comportamento dos consumidores de vinhos com indicação geográfica. Além disso, cada vez mais os consumidores desenvolvem a percepção de qualidade em relação aos vinhos com indicação geográfica, pelo fato de essas certificações transmitirem confiabilidade aos produtos.

Os resultados indicam que os vinhos com indicação geográfica estão 
associados a atributos locais que remetem à qualidade sensorial, tipicidade e prestígio, capazes de gerar um diferencial competitivo no mercado. Pode-se inferir, assim, que existe um cenário otimista para o mercado de vinhos com indicação geográfica, oportunizando o desenvolvimento de mercados em países ainda sem tradição consolidada na produção de vinhos.

A partir dos resultados, identifica-se um cenário vitícola global propício para o consumo de vinhos com indicação geográfica, exigindo das vinícolas a utilização de diferentes estratégias para a diferenciação e valorização de seus produtos, principalmente aqueles relacionados aos atributos regionais. Conclui-se, ainda, ao pensar no que influencia a decisão de compra do consumidor, que os atributos intrínsecos e extrínsecos estão vinculados e representam uma determinada região, mesmo que não haja a distinção formal de indicação geográfica.

Como principais implicações desse estudo para o meio acadêmico, destacase a utilização de uma metodologia científica capaz de reproduzir resultados de diferentes estudos a partir de um protocolo específico. A partir de uma revisão sistemática da literatura foi possível a utilização de estudos primários e teóricos que contemplaram o tema. Futuras pesquisas podem utilizar outros protocolos para identificar diferentes estudos a fim de compreender as variáveis que interferem na percepção de qualidade dos consumidores de vinhos com indicação geográfica ou com atributos regionais. Dessa forma seria possível comparar com os resultados aqui encontrados, a fim de verificar convergências e divergências nos resultados apresentados pelos estudos.

\section{AGRADECIMENTOS}

Ao Instituto Federal de Educação, Ciência e Tecnologia do Rio Grande do Sul (IFRS), pelo financiamento, em decorrência da atuação do primeiro dos autores do artigo na Instituição. Os autores agradecem, igualmente, ao Centro de estudos e Pesquisas em Agronegócios (Cepan) da Universidade Federal do Rio Grande do Sul (UFRGS), pelo apoio na elaboração da pesquisa. 


\section{REFERÊNCIAS}

ADINOLFI, F.; De ROSA, M.; TRABALZI, F. Dedicated and generic marketing strategies The disconnection between geographical indications and consumer behavior in Italy. British Food Journal, v. 113, n. 2-3, p. 419-435, 2011.

ALBERSMEIER, F.; SCHUlZE, H.; JAHN, G.; SPILLER, A. The reliability of thirdparty certification in the food chain: from checklists to risk-oriented auditing. Food Control, v. 20, n. 10, p. 927-935, 2009.

ALEBAKI, M.; IAKOVIDOU, O. Segmenting the greek wine tourism market using a motivational approach. New Medit, v. 9, n. 4, p. 31-40, 2010.

ATKIN, T.; THACH, L. Millennial wine consumers: risk perception and information search. Wine Economics and Policy, v. 1, n. 1, p. 54-62, 2012.

AZABAGAOGLU, M. O.; AKYOL, A.; OZAY, A. Examining the turkish wine industry: marketing effectiveness and recommendations for increasing its competitive performance. New Zealand Journal of Crop and Horticultural Science, v. 34, n. 3, p. 257-268, 2006.

BALESTRINI, P.; GAMBLE, P. Country-of-origin effects on chinese wine consumers. British Food Journal, v. 108, n. 5, p. 396-412, 2006.

BERNABÉU, R.; PRIETO, A.; DÍAZ, M. Preference patterns for wine consumption in Spain depending on the degree of consumer ethnocentrism. Food Quality and Preference, v. 28, n. 1, p. 77-84, 2013.

BROWN, G.; GETZ, D. Linking wine preferences to the choice of wine tourism destinations. Journal of Travel Research,v. 43, n. 3, p. 266-276, 2005.

BRUWER, B.; BURROWS, N.; CHAUMONT, S.; LI, E.; SALIBA, A. Consumer involvement and associated behaviour in the UK high-end retail off-trade wine market. The International Review of Retail, Distribution and Consumer Research. 24 p. 145-165, 2014. 
BRUWER, J.; BULLER, C. Product involvement, brand loyalty, and country-of-origin brand preferences of Japanese wine consumers. Journal of Wine Research, 24: p. 38-58, 2013.

BRUWER, J.; SALIBA, A.; MILLER, B. Consumer behaviour and sensory preference differences: implications for wine product marketing. Journal of Consumer Marketing, v. 28, n. 1, p. 5-18, 2011.

BRUWER, J.; LESSCHAEVE, I.; CAMPBELL, B. L. Consumption dynamics and demographics of canadian wine consumers: retailing insights from the tasting room channel. Journal of Retailing and Consumer Services, v. 19, n. 1, p. 45-58, 2012.

CACIC, J.; TRATNIK, M.; KLJUSURIC, J. G.; CACIC, D.; KOVACEVIC, D. Wine with geographical indication - awareness of croatian consumers. British Food Journal, v. 113 , n. 1, p. 66-77, 2011.

CANIGLIA, E.; D'AMICO, M.; PERI, I. An analysis of consumers' perception of the quality of the Etna DOC wine. New Medit, v. 7, n. 3, p. 32-40, 2006.

CHREA, C.; MELO, L.; EVANS, G.; FORDE, C.; DELAHUNTY, C.; COX, D. N. An investigation using three approaches to understand the influence of extrinsic product cues on consumer behavior: an example of australian wines. Journal of Sensory Studies, v. 26, n. 1, p. 13-24, 2011.

CHRYSOCHOU, P.; CORSI, A. M.; KRYSTALLIS, A. What drives greek consumer preferences for cask wine? British Food Journal, v. 114, n. 8-9, p. 1072-1084, 2012.

COLICCHIA, C.; STROZZI, F. Supply chain risk management: a new methodology for a systematic literature review. Supply Chain Management-an International Journal, v. 17, n. 4, p. 403-418, 2012.

CORDUAS, M.; CINQUANTA, L.; IEVOLI, C. The importance of wine attributes for purchase decisions: a study of italian consumers' perception. Food Quality and Preference, v. 28, n. 2, p. 407-418, 2013. 
FAO. Food and Agriculture Organization. Principles for responsible agricultural investment that respects rights, livelihoods and resources. Extended version. Rome, 2010.

GARCIA-GALAN, M. M.; Del MORAL-AGUNDEZ, A.; GALERA-CASQUET, C. Valuation and importance of the extrinsic attributes of the product from the firms' perspective in a spanish wine protected designation of origin. Spanish Journal of Agricultural Research, v. 12, n. 3, p. 568-579, 2014.

GJONBALAJ, M. et al. Analyses of consumer behavior and wine market in Kosovo. Agriculturae Conspectus Scientificus,v. 74, n. 4, p. 333-338, 2009.

GREENHALGH T. Papers that summarise other papers (systematic review and metaanalyses). BMJ, v. 13, n. 315, p. 672-675, sep. 1997.

HAWKINS, D. I.; MOTHERSBAUGH, D. L. Consumer behaviour: building marketing strategy. 12.ed. New York, USA: McGraw-Hill, Irwin, 2012.

JAEGER, S. R.; DANAHER, P. J.; BRODIE, R. J. Wine purchase decisions and consumption behaviours: insights from a probability sample drawn in Auckland, New Zealand. Food Quality and Preference, v. 20, n. 4, p. 312-319, 2009.

KaLLAS, Z.; ESCOBAR, C.; GIL, J. M. Analysis of consumers' preferences for a specialoccasion red wine: a dual response choice experiment approach. Food Quality and Preference, v. 30, n. 2, p. 156-168, 2013.

KRYSTALLIS, A.; CHRYSOCHOU, P. An exploration of loyalty determinants in greek wine varieties. Managerial and Entrepreneurial Developments in the Mediterranean Area, p. 1004-1013, 2009.

LOCKSHIN, L. S.; SPAWTON, A. L. Using involvement and brand equity to development a wine tourism strategy. International Journal of Wine Marketing, v. 13,n. 1, p. 72-81, 2001.

MILOHANOVIĆ, A.; BRŠČIĆ, K. Tourists perceptions of istrian wines. Agriculturae Conspectus Scientificus, v. 73, n. 2, p. 121-125, 2008. 
MUELLER, S.; SZOLNOKI, G. The relative influence of packaging, labelling, branding and sensory attributes on liking and purchase intent: consumers differ in their responsiveness. Food Quality and Preference, v. 21, n. 7, p. 774-783, 2010.

NDANGA, L. Z. B.; LOUW, A.; Van ROOYEN, J. Increasing domestic consumption of south african wines: exploring the market potential of the "black diamonds". Agrekon, v. 49, n. 3, p. 293-315, 2008.

OIV. International Organization of Vine and Wine, 2014.

OLSON, J. C.; JACOBY, J. Cue utilization in the quality perception process. In: Conference of the association for consumer research, v. 3., p. 79-167. Iowa City, 1972.

PERROUTY, J. P.; D'HAUTEVILLE, F.; LOCKSHIN, L. The influence of wine attributes on region of origin equity: an analysis of the moderating effect of consumer's perceived expertise. Agribusiness, v. 22, n. 3, p. 323-341, 2006.

PRIILAID, D.; Van RENSBURG, P. The hedonic valuation of South African wine brands. South African Journal of Business Management, v. 43, n. 1, p. 11-31, 2012.

RODRÍGUEZ-SANTOS, M. C.; GONZÁLEZ-FERNÁNDEZ, A. M.; CERVANTES-BLANCO, $\mathrm{M}$. An analysis of the construct "involvement" in consumer behaviour. Quality and Quantity, v. 47, n. 2, p. 1105-1123, 2013.

ROMA, P.; MARTINO, G. D.; PERRONE, G. What to show on the wine labels: a hedonic analysis of price drivers of Sicilian wines. Applied Econimics, p. 2765-2778, 2013.

ROSSI, M. Competitive strategy and performance of campania wine firms. Managerial and Entrepreneurial Developments in the Mediterranean Area, p. 1556-1575, 2009.

SAENZ-NAVAJAS, M. P.; BALLESTER, J.; PEYRON, D.; VALENTIN, D. Extrinsic attributes responsible for red wine quality perception: a cross-cultural study between France and Spain. Food Quality and Preference, v. 35, p. 70-85, 2014. 
SCHIFFMAN, L. G. Consumer behavior. Englewood Cliffs: Prentice Hall, 1997.

SKURAS, D.; DIMARA, E. Regional image and the consumption of regionally denominated products. Urban Studies,v. 41, n. 4, p. 801-815, 2004.

THIENE, M.; SCARPA, R.; GALLETTO, L.; BOATTO, V. Sparkling wine choice from supermarket shelves: the impact of certification of origin and production practices. Agricultural Economics, v. 44, n. 4-5, p. 523-536, 2013.

TSAPI, V.; DJEUMENE, P.; TCHUENTE, M. Rôle du pays d'origine dans la perception de la qualité du vin par le consommateur aficain: une étude menée dans le contexte camerounais. Market Management, v. 9, 2009. 136p.

URSO, J. J. Stress e personalidade: "overview" e avaliação crítica de revisões sistemáticas sobre padrão comportamental tipo A e personalidade tipo D com desfechoscoronarianos. 2011. Tese (Doutorado)-Instituto de Psicologia, Universidade de São Paulo - USP, 2011.

Recebido em: 07/12/2015

Aceito em: 20/04/2017 